\title{
Dynamics and Growth of Droplets Close to the Two-Phase Coexistence Curve in Fluids
}

\author{
Sutapa Roy and Subir K. Das* \\ Theoretical Sciences Unit, Jawaharlal Nehru Centre for Advanced Scientific Research, Jakkur P.O, Bangalore 560064, India
}

(Dated: June 27, 2018)

\begin{abstract}
Results from the state-of-the-art molecular dynamics simulations are presented for both equilibrium and nonequilibrium dynamics following vapor-liquid transition in a single component LennardJones system. We have fixed the overall density close to the vapor-branch of the coexistence curve so that the liquid phase forms droplet structure in the background of vapor phase. In the equilibrium case, the motion of a single droplet is studied in both microcanonical and canonical ensembles, in the latter case a hydrodynamics preserving Nosé-Hoover thermostat was used to control the temperature. The droplet nucleation, motion, collision and coalescence dynamics in the nonequilibrium case were studied in the canonical ensemble with Nosé-Hoover thermostat. There it was observed that the average droplet volume grows linearly with time. Between two successive collisions, the size of the droplets remain same even though all the constituent particles do not move with the droplets - some leave, others join. It is seen that the number of original particles in a droplet decays exponentially fast. Results from a liquid-liquid transition are also presented in the equilibrium context. Dynamics of droplets in equilibrium appears to be at variant with the nonequilibrium case.
\end{abstract}

\section{INTRODUCTION}

The subject of nucleation remains interesting over many decades [1 5]. Physics of nucleation and subsequent growth is certainly challenging and answers to many fundamental questions in this branch are subjects of debate. On the other hand, understanding of these phenomena is crucial to many practical applications [6 6 ] including nanoscience and technology.

When a homogeneous system is quenched (say, by sudden change of temperature $(T)$ ) inside the coexistence curve the system phase separates into particle rich and particle poor domains 9 -12]. While for quenches close to the critical density (or composition in a binary system) the phase separation or domain coarsening is spontaneous (referred to as spinodal decomposition), for ones close to the coexistence curve the system may reside in a metastable state in which case phase separation starts only after nucleation of droplets following rare long wave length fluctuations 3 .

In this work, staying outside the so-called spinodal regime, we address the issue of dynamics of fluid droplets and their growth, once they are formed. Results are presented for both equilibrium and nonequilibrium situations, for a vapor-liquid as well as a liquid-liquid transition. For the liquid-liquid case, however, because of the computational demand, we will confine ourselves to studies in equilibrium condition. The primary emphasis in this paper being on the nonequilibrium dynamics 9 -12], below we briefly review the subject to place our work in appropriate perspectives. Note here that we expect the discussions provided below to be valid for both liquidliquid and vapor-liquid transitions, even though some of them were originally meant for liquid-liquid cases or binary mixtures in general.

Coarsening of domains in solid binary mixtures is due to diffusive transport and follows the celebrated LifshitzSlyozov (LS) law [9, 13 15], where the average length, $\ell(t)$, of domains grows with time $(t)$ as $t^{1 / 3}$. This scaling law is true irrespective of a quench close to the critical composition or near the coexistence curve. On the other hand, in fluids, hydrodynamics plays important role 16
21]. There, for quenches close to the critical density or composition, where one has an interconnected domain structure, one expects three different regimes of growth. The early time diffusive LS mechanism is followed by a linear viscous [9, 10, 17 21] hydrodynamic regime with an exponent $\alpha=1$ and further by an inertial [9, 10, 19, 20] hydrodynamic regime with an exponent $2 / 3$. It is argued that through the tube-like interconnected domains material flow becomes faster due to hydrodynamics. On the other hand, one has a disconnected droplet morphology close to the coexistence curve where the domains are expected to grow via collisions [21 30].

There are different possibilities for the mechanism of the above mentioned collision events. If there exists any inter-droplet force [24, 25, 27, 28], the dynamics of droplets is going to be deterministic. Other possibility is that the droplets exhibit Brownian motion and collide randomly 21 23]. Even though both the cases are discussed in the literature, the dynamics of coarsening of liquid droplets received much less attention as opposed to spinodal decomposition. In this work our primary focus is on the late stages of droplet growth and understanding the microscopic details of the motion of the droplets. Some preliminary results in this context were published in a recent communication [29].

According to Binder and Stauffer (BS) [21 23], if the motion of the droplets is Brownian, $\ell(t)$ should grow as $t^{1 / 3}$. In that case, one can write the equation for the rate of change of droplet density $n$ as

$$
\frac{d n}{d t}=C_{1} D_{d} \ell n^{2}
$$

where $D_{d}$ is the droplet diffusivity and $C_{1}$ is a constant. Using $n \propto 1 / \ell^{3}$ and noting that, according to StokesEinstein-Sutherland (SES) relation [16, 31, 32], $D_{d} \ell$ is a constant, solution of Eq.(1) gives

$$
\ell(t) \sim t^{1 / 3}
$$

Tanaka proposed that based on the density of droplets, the motion could be deterministic [24, 25]. He argued, the BS mechanism will be valid only in the low droplet density limit, for minority species (in a binary mixture 
context) volume fraction $\phi<0.06$. Then the interdroplet distance is much larger than $\ell$ such that the dropletdroplet interaction can be neglected. In such situations the droplets can freely undergo Brownian motion and random collisions. On the other hand, at high droplet density (with $\phi>0.06$ ), the diffusion field or the concentration gradients around neighbouring droplets get coupled which gives rise to an attractive interaction between the droplets 24 26]. This attractive force eventually induces a direct collision. This can also be appreciated by considering the excess free energy $\sim(\nabla \psi)^{2}$ coming from the concentration (or density) gradient, $\psi$ being the concentration. The excess energy provides a drive for removing the gradient zone, thus causing the attractive interaction between the neighbouring droplets. Note that this gradient term gives rise to the diffusion under concentration gradient in Ginzburg-Landau formalism [9, 10, 12]. This sort of interdroplet interaction will surely accelerate the droplet growth mechanism. However, for the form of interaction obtained by Tanaka, this deterministic motion of droplets provides the exponent for the growth law same as the Brownian mechanism but a higher amplitude which, for the sake of brevity, we do not discuss here. The brief discussion below, however, provides idea of how to obtain some knowledge about it from computer simulations.

The ratio of the growth amplitudes $A_{L S}$ and $A_{B S}$ in the LS and BS mechanisms has been pointed out to be [21, 24, 25]

$$
A_{B S} / A_{L S}=K \phi^{1 / 3} ; K \simeq 6 .
$$

It has also been argued [24, 25] that $K \simeq 4.84$ due to some ambiguity in the value of constant $C_{1}$ in Eq. (1). By incorporating appropriate dynamics, in computer simulations, it is possible to estimate this ratio. This, in turn, will provide the realization of actual mechanism, Brownian or non-Brownian. We will use this fact plus other means to figure out the presence of inter-droplet interaction.

In this work, in addition to estimating the exponent of the fluid droplet growth, we investigate the true mechanism for the growth. Further, we aim to gain knowledge about the microscopic origin of droplet motion in both equilibrium and nonequilibrium contexts, validity of SES relation for these objects, etc. Number of results we have presented here and methodologies used for that purpose are nonconventional in the context of coarsening dynamics.

The rest of the paper is organized as follows. In Sec. II we discuss the model and the methodologies. Results are presented in Sec. III. Finally, Sec. IV concludes the paper with an outlook for some of the future possibilities.

\section{MODELS AND METHODS}

For this study, we choose a model in which particles of equal mass $(m)$, at distance $r$ apart, interact via [18, 29]

$u\left(r=\left|\vec{r}_{i}-\vec{r}_{j}\right|\right)=U(r)-U\left(r_{c}\right)-\left(r-r_{c}\right)(d U / d r)_{r=r_{c}},(4)$ where, for the vapor-liquid transition,

$$
U(r)=4 \varepsilon\left[\left(\sigma / r_{i j}\right)^{12}-\left(\sigma / r_{i j}\right)^{6}\right]
$$

is the standard Lennard-Jones (LJ) pair potential, $r_{c}$ is a cut-off distance for the LJ potential (introduced for faster computation), $\sigma$ is the particle diameter and $\varepsilon$ is the interaction strength. For the present work, we have set $r_{c}=2.5 \sigma$. The model exhibits a gas-liquid phase transition [29] with critical temperature $k_{B} T_{c} \simeq 0.9 \varepsilon$ and critical density $\rho_{c} \simeq 0.3$. Note that $\rho$ is defined as $N \sigma^{3} / L^{3}$ where $N$ is the number of particles in a cubic box of linear dimension $L$ in units of $\sigma$. For the rest of the paper, we set $\varepsilon=1, m=1, \sigma=1$ and the Boltzmann constant $k_{B}=1$. This sets the LJ time unit $\sqrt{m \sigma^{2} / \varepsilon}=1$.

The nonequilibrium dynamics using this model is studied via the molecular dynamics (MD) [33, 34] simulations in NVT ensemble using two different thermostats to control the temperature. To probe the hydrodynamic effects we have used the Nosé-Hoover thermostat (NHT) 33, 35. On the otherhand, an Andersen thermostat (AT) 33] was used to study the difference between a growth via the droplet diffusion mechanism, studied via NHT, and the simple LS mechanism. We have also used the perfectly hydrodynamics preserving NVE ensemble to probe the dynamics of a single droplet in the equilibrium situation. Even though this latter ensemble preserves hydrodynamics perfectly, it cannot be used for the nonequilibrium study since it cannot keep the temperature constant during the coarsening process.

The time step for the MD runs was chosen to be $\Delta t=0.005$ in the Verlet velocity algorithm 33]. We applied periodic boundary conditions in all directions. All results correspond to $T=0.6$ and $\rho=0.05$. Note that at this temperature the coexistence vapor density is $\simeq 0.01$. Unless otherwise mentioned, all results for the nonequilibrium case, obtained using the NHT, are averaged over 12 independent initial configurations, with $L=100$.

At $t=0$, initial configurations with homogeneous density, prepared at a high temperature $T=5$, were quenched to $T=0.6$ inside the coexistence curve. To understand the subsequent domain growth and related dynamics, we computed several important quantities which we discuss below.

The average domain size, $\ell(t)$, was calculated using the following scaling property of the two-point equal time order-parameter correlation function, $C(r, t),(r$ being the spatial separation between two points) [9]

$$
C(r, t) \equiv \tilde{C}(r / \ell(t)) .
$$

To facilitate the calculation of $C(r, t)$, we have mapped [14, 15, 36] a continuum fluid system into a simple cubic lattice of lattice constant $\sigma$, where particles in the actual system were moved to the nearest lattice sites. In the mapped system, a site, with index $i$, occupied by a particle was assigned a spin value $S_{i}=+1$, otherwise -1 . Note that in this spin language the value of $\phi$, the volume fraction of the minority species (in this case the up spins), appears to be 0.054 . Finally, $C(r, t)$ was calculated from the standard formula $C(r, t)=\left\langle S_{i} S_{j}\right\rangle-\left\langle S_{i}\right\rangle\left\langle S_{j}\right\rangle$, $r=|\vec{i}-\vec{j}|$. Further, $\ell(t)$ was also calculated directly by sweeping through the lattice and identifying the domain boundaries. Another important quantity, may be difficult to calculate, that gives information about $\ell(t)$ is the number of particles in droplets, $N_{p}$, since $\left\langle N_{p}(t)\right\rangle \propto \ell(t)^{3}$. The distribution function of $N_{p}$ should obey the scaling 


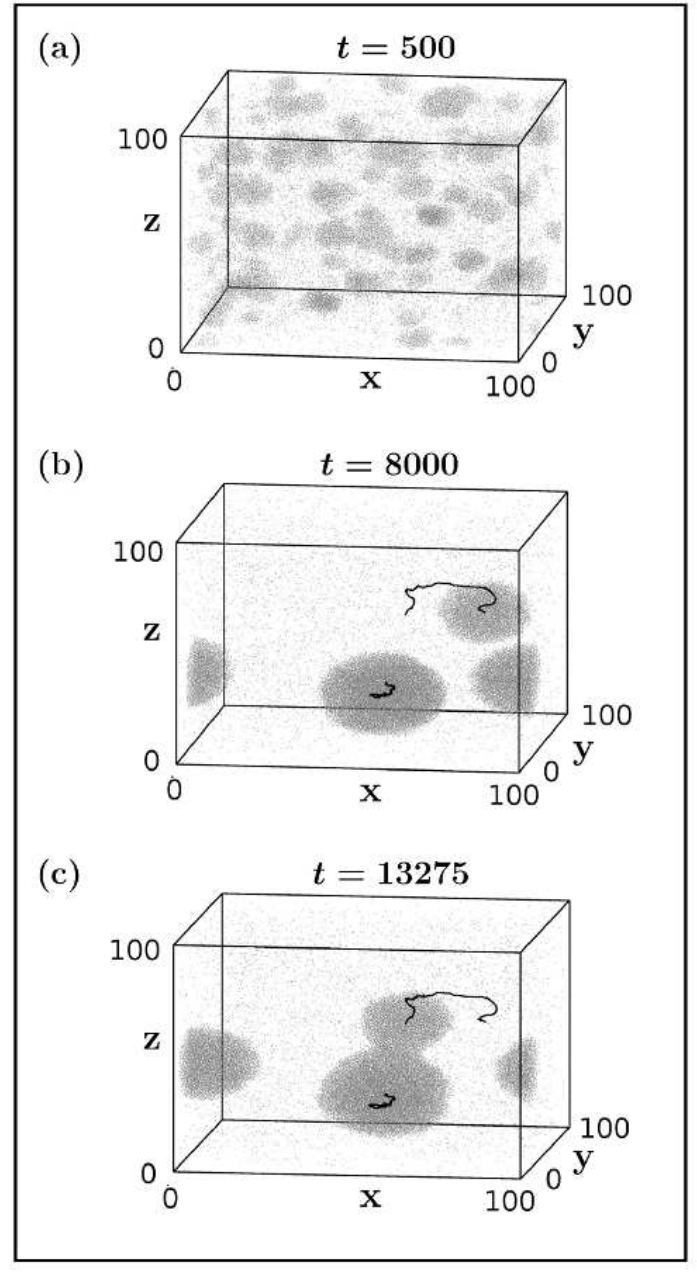

FIG. 1. Evolution snapshots following a temperature quench of a low density $(\rho=0.05)$ homogeneous fluid, prepared at a high temperature, to a temperature $T=0.6$, inside the coexistence curve. The zig-zag lines in (b) and (c) are the trajectories of two droplets during the interval between $t=$ 8000 and $t=13275$.

relation

$$
P\left(N_{p}, t\right) \equiv \frac{1}{\left\langle N_{p}\right\rangle} \tilde{P}\left(N_{p} /\left\langle N_{p}\right\rangle\right)
$$

In Eqs. (6) and (7), $\tilde{C}$ and $\tilde{P}$ are master functions independent of time.

To calculate $N_{p}$, one first needs to identify the droplets for which we used the following method. We calculated the density surrounding each particle, by defining a neighbourhood of radius $R$, using the continuum configuration and if the density was higher than the critical value, the particle was marked as a constituent of any of the droplets. Note that this radius cannot be too small or too large. In the limiting case $R=$ the particle radius, one is not including any neighbour. On the otherhand, for large value of $R$, there is the risk of moving to a different phase if the central particle is close enough to the interface. We have taken care of these facts. Next, depending on the spatial separations between these marked particles, different droplets were identified and the particles were indexed according to their parent droplets. Following this, information about $N_{p}$, droplet diameter $\left(d_{d}\right)$,

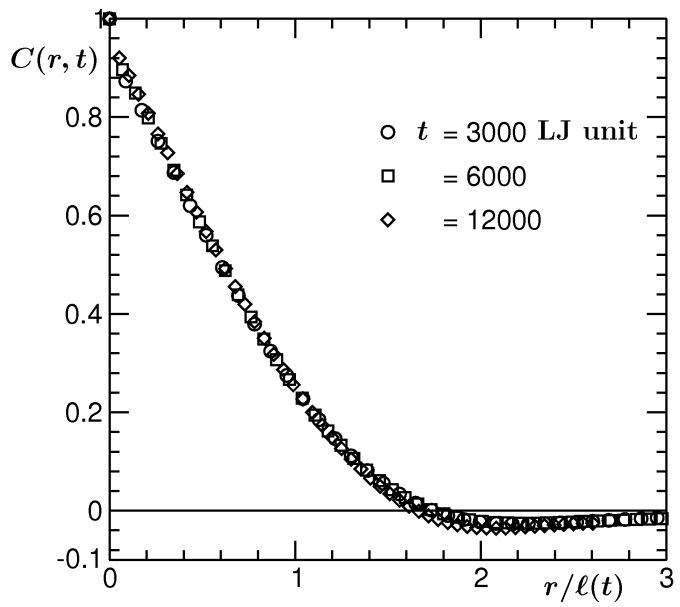

FIG. 2. Scaling plot of the correlation function $C(r, t)$ as a function of $r / \ell(t)$. Data from three different times are used. The values of $\ell(t)$ were obtained from the decay of $C(r, t)$ to $1 / 4$ th its maximum value. The results correspond to $\rho=0.05$, $L=100$ and $T=0.6$ and an averaging over 12 independent initial configurations.

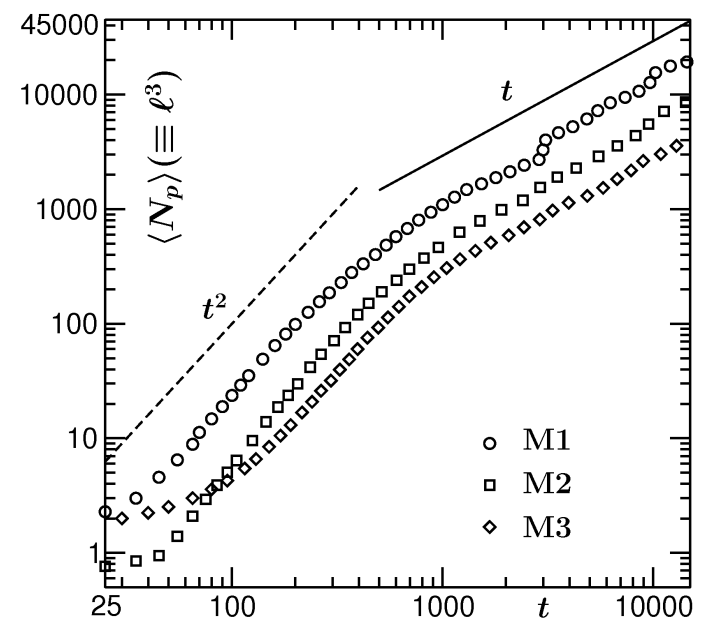

FIG. 3. Log-log plots of the average number of particles in droplets, $\left\langle N_{p}\right\rangle$, or the average droplet volume, $\ell^{3}$, as a function of time, during the nonequilibrium evolution, calculated via three different methods. Possibilities for various different power laws are indicated.

total number of droplets $\left(N_{d}\right)$ in the system at time $t$, etc., can be obtained in a straight forward manner. Note that to obtain exact information about $d_{d}$ (most accurate measure of $\ell$ ), we need to calculate the density inside the droplet also.

In order to study the nature of droplet motion, we calculated the mean squared displacement (MSD) of the centre of mass $(\mathrm{CM})$ of a droplet as [16]

$$
M S D=\left\langle\left(\vec{R}_{C M}(t)-\vec{R}_{C M}(0)\right)^{2}\right\rangle,
$$

where the position vector of the droplet $\mathrm{CM}, \vec{R}_{C M}$, has 


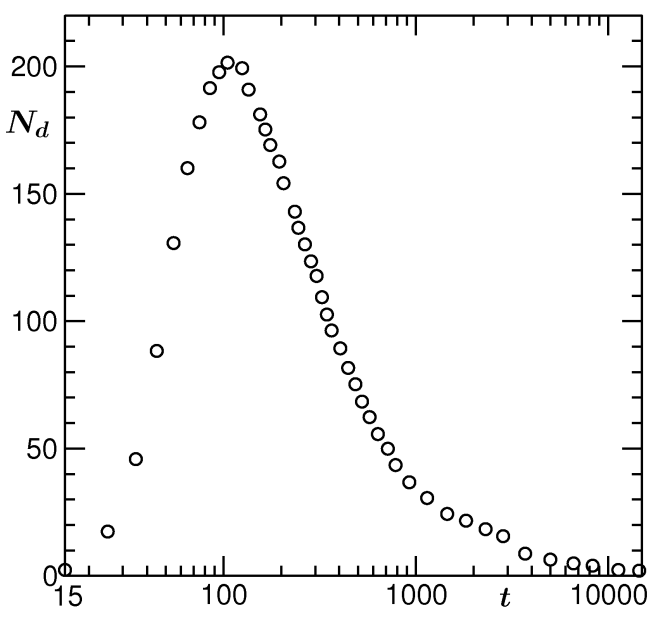

FIG. 4. Semi-log plot of the average number of droplets, $N_{d}$, as a function of time. All the system parameters are same as the previous figures.

the standard definition

$$
\vec{R}_{C M}(t)=\frac{1}{N_{p}} \sum_{i=1}^{N_{p}} \vec{r}_{i}(t) .
$$

According to our observation, as the droplets move, the constituent particles also keep changing - some leave, some join. Therefore, while calculating the MSD, at each instant particles within the droplet were identified and $\vec{R}_{C M}$ at that instant was calculated using those particles only. The diffusivity, $D_{d}$, of a droplet was calculated using the relation [16]

$$
D_{d}(t)=\lim _{t \rightarrow \infty} \frac{M S D}{6 t},
$$

and verified if $D_{d}$ follows the SES relation [16, 31, 32]

$$
D_{d} \sim \frac{T}{6 \pi \eta d_{d}}
$$

$\eta$ being the effective shear viscosity of the background fluid. As we will see, it appears that this holds well in the equilibrium situation for both vapor-liquid and liquidliquid transitions. For the latter, we briefly discuss the model below.

In the liquid-liquid $(A+B)$ case, we choose a symmetric model with the same interaction (4) but the LJ potential given as

$$
U\left(r=\left|\vec{r}_{i}-\vec{r}_{j}\right|\right)=4 \varepsilon_{i j}\left[\left(\frac{\sigma_{i j}}{r_{i j}}\right)^{12}-\left(\frac{\sigma_{i j}}{r_{i j}}\right)^{6}\right],
$$

with

$$
\begin{gathered}
\sigma_{A A}=\sigma_{B B}=\sigma_{A B}=\sigma, \\
\varepsilon_{A A}=\varepsilon_{B B}=2 \varepsilon_{A B}=\varepsilon .
\end{gathered}
$$

We set the overall density of the system to unity. More details on this model and related phase diagram can be found in Refs. [37, 38]. We just mention here that this binary mixture has $T_{c} \simeq 1.42$ and because of the symmetry in Eq. (13), the critical composition is $50 \% A$ and
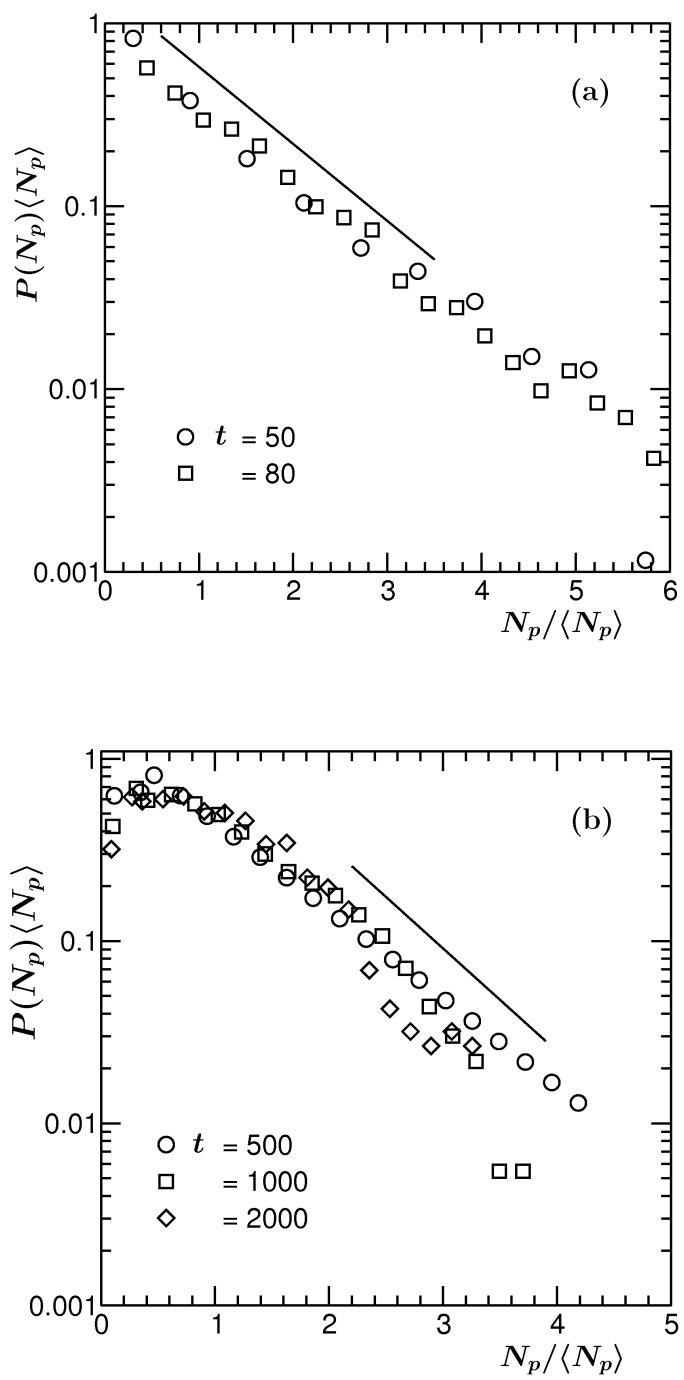

FIG. 5. Semi-log plots of the scaled distribution functions of the number of particles in droplets $\left(N_{p}\right)$, from different times. The solid lines corresponds to exponential decay. (a) During droplet nucleation stage. (b) During droplet growth stage.

$50 \% B$. In this work, the concentration of $A$ species, $x_{A}\left(=\frac{N_{A}}{N}, N_{A}\right.$ and $N$ being respectively the number of $A$ particles and total number of particles), was set to 0.15 and temperature to $T=1.0$. For this choice of parameters, droplets of $A$ species are formed in the background of $B$. All results for the binary fluid are obtained from 8 independent initial configurations in NVE as well as NVT (NHT) ensembles. Periodic boundary conditions were applied in all directions. In this case the droplets were identified following a similar procedure as in the vapor-liquid case, considering the $A$ particles only. Subsequently the droplet MSD and droplet diffusivity were calculated using Eq. (8) and Eq. (10) respectively.

Next we move on to present the results. Unless otherwise mentioned, all results correspond to the vapor-liquid transition. 


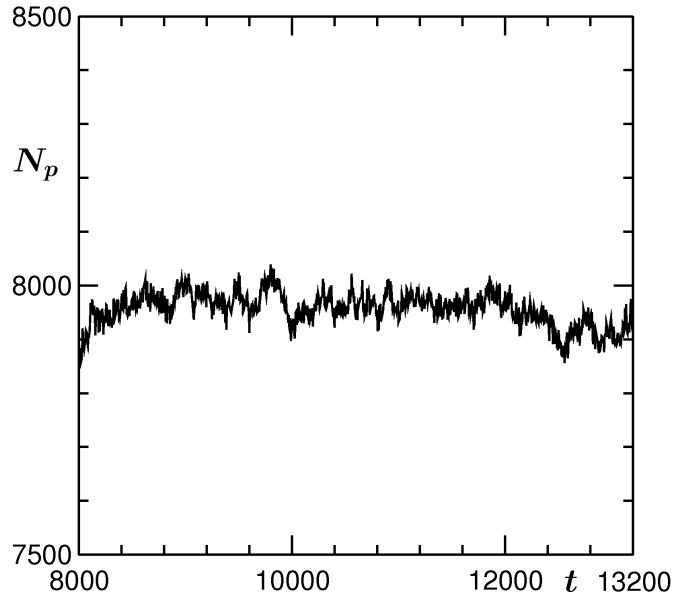

FIG. 6. Plot of the number of particles in a typical droplet before it undergoes a collision.

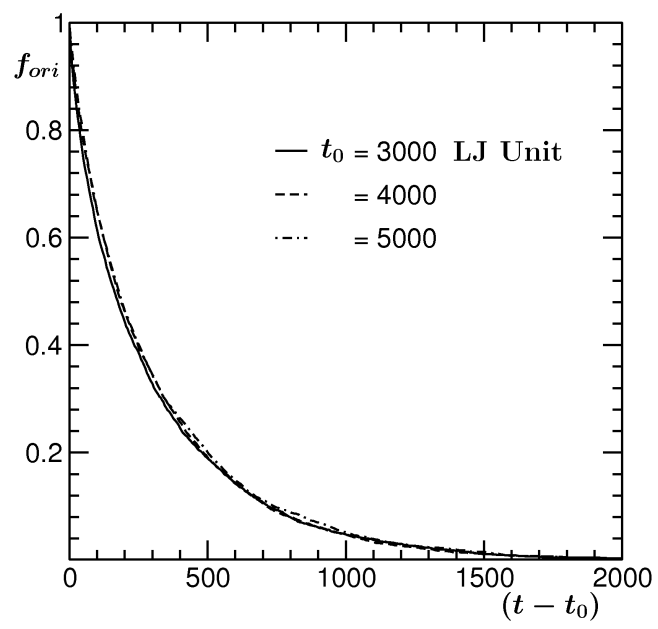

FIG. 7. Plots of the fraction, $f_{\text {ori }}$, of original particles at time $t_{0}$, that move with the droplet, as a function of $\left(t-t_{0}\right)$. Results for three different values of $t_{0}$ are shown.

\section{RESULTS}

In Fig. 1, we show the snapshots during the nonequilibrium evolution of a vapor-liquid system at $T=0.6$, starting from a homogeneous density, prepared at a high temperature. Already at $t=500$, there are reasonably well formed droplets in the system. The other two snapshots in (b) and (c) demonstrate the growth of the droplets with time. In (b) and (c) we have also drawn the trajectories of two droplets starting from $t=8000$ to 13275 . Note that the droplets collide with each other at $t=13275$. While the trajectories certainly have some degree of randomness, one needs further probes to confirm whether they are truly Brownian or not. A look at the mean squared displacements of the droplets should prove useful, to which we will come back later.

In Fig. 2 we plot $C(r, t)$ vs the scaled distance $r / \ell(t)$ for three different times. Note that $\ell(t)$ used here was obtained from the decay of the $C(r, t)$ to $1 / 4$ th its maximum value. The excellent quality of data collapse indi- cates self-similarity of the structures in this time regime.

Fig. 3 shows the plots of the average number of particles in droplets, $\left\langle N_{p}\right\rangle$, and the average droplet volume, $\ell(t)^{3}$, vs $t, \ell(t)$ being calculated via different methods. Double$\log$ scale is used. Note that $\left\langle N_{p}\right\rangle \propto \ell(t)^{3}$ holds under the assumption that the density in the liquid droplets does not change over time. We calculated $N_{p}$ directly from the knowledge of the constituent particles of droplets (M1), $\ell(t)$ from the decay of the correlation functions (M2) and by the method of sweeping through the lattice and identifying the domain interfaces (M3). All these were discussed in the previous section. The assumption of constant density of droplets does not hold at early time. Because of this there is some qualitative discrepancy of measure M1 with the other ones at early time. The solid lines in this plot correspond to different possible powerlaw scaling regimes. Following an initial faster growing regime with an exponent $2 / 3$, the system enters into a regime where $\ell(t) \sim t^{1 / 3}$. This latter regime may be due to the Brownian droplet diffusion and collision mechanism of BS 22]. Certainly the very nice consistency of all the three data sets with the $t^{1 / 3}$ behavior confirms the growth exponent predicted by BS, if not the mechanism. Alongside, it also validates our different methods of identifying $\ell$. A discussion of the possibility of a $t^{2 / 3}$ behavior, at early time, is provided in Ref.[29]. Possibility of a linear growth in this regime can also not be ruled out. This is considering the fact that a crossover is never very sharp and what appears as $2 / 3$ may well be due to a gradual crossover from linear to $1 / 3$ law. Possibility of a linear behavior was discussed by Tanaka and not an objective of this paper.

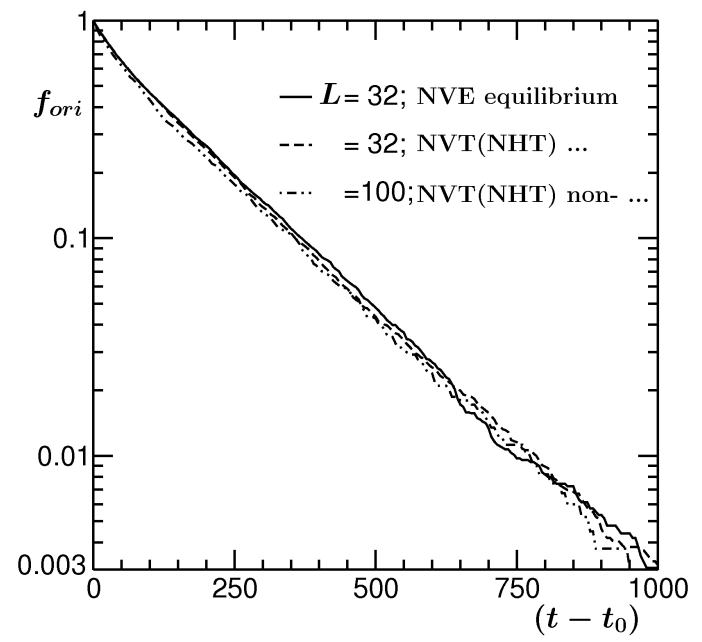

FIG. 8. Plots of $f_{\text {ori }}$ as a function of $t-t_{0}$. Results are presented for three different cases: equilibrium situation (only one droplet present) in the NVE ensemble as well as with NHT and the nonequilibrium situation with the NHT. In all the cases the sizes of the droplets are nearly the same. A semi-log scale is used.

In Fig. 4 we plot the average number of droplets in the system, $N_{d}$, vs $t$, on a log-linear scale. As observed, beyond a time $t_{g}=t=115, N_{d}$ decays due to droplet coalescence (during which the average domain size increases, see Fig. 3). During time before $t_{g}$, mostly droplets keep 

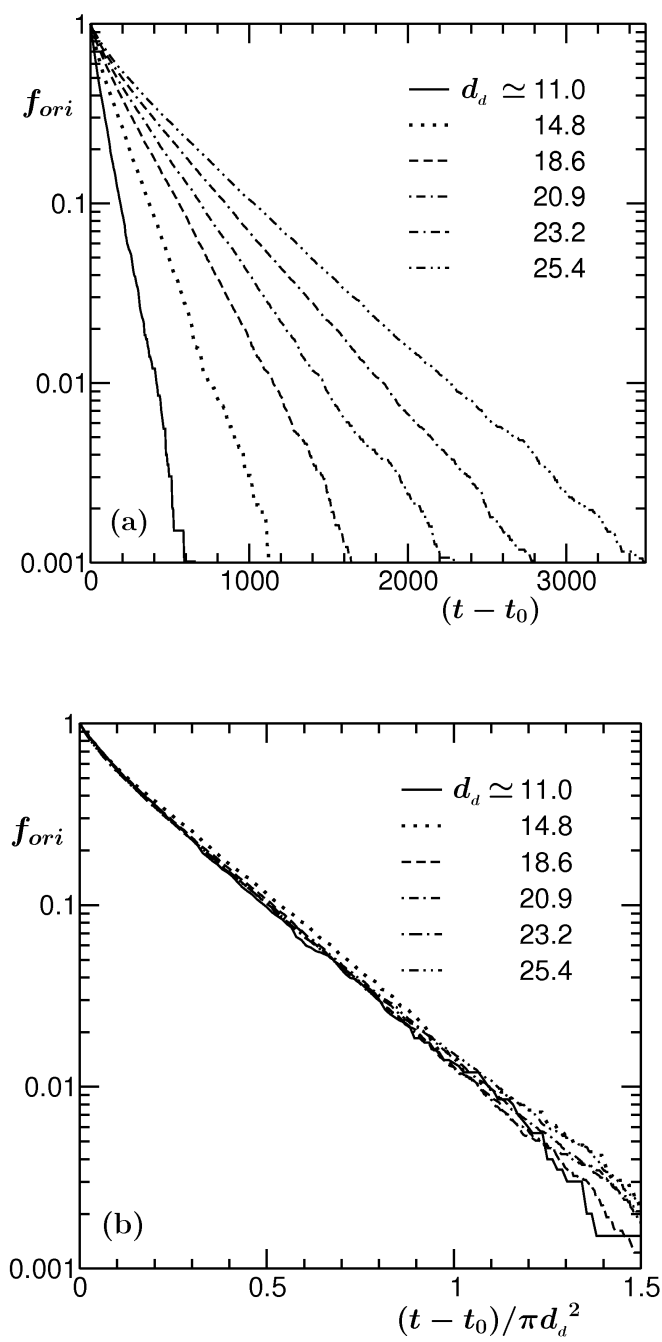

FIG. 9. (a) Plots of $f_{\text {ori }}$ as a function of $t-t_{0}$ for different droplet sizes, obtained from equilibrium configurations using NVE ensemble. (b) Same as (a), but the $x$ axis scaled by $\pi d_{d}^{2}$.

forming. It will be interesting to study how in these two different regimes the probability distributions of $N_{p}$, $P\left(N_{p}, t\right)$, compare. In Figs. [5(a) and (b) we show the scaling plots of the distribution functions [see Eq. (7)] using data from different times, as indicated in the figure, on a semi-log scale. Part (a) here is for $t<t_{g}$ and (b) for $t>t_{g}$. The solid lines there correspond to exponential decay. In the large droplet limit the distributions from both the time regimes are consistent with exponential decay, but difference exists in the small droplet size regime. Fast exponential decay in Fig. 5(a) from the very beginning is suggestive that all the droplets are roughly of same size which is expected at the formation stage. On the other hand, when the droplets are growing, there must be dispersion in size. This is reflected in Fig. [5(b) where for small values of the scaling variable $N_{p} /\left\langle N_{p}\right\rangle$, the distribution has a nearly flat character. This result is in agreement with previous studies [15, 18], where instead of the domain volumes, distribution of domain lengths were studied.

Next we move on to discuss the droplet growth mechanism. We will be dealing only with the late stage
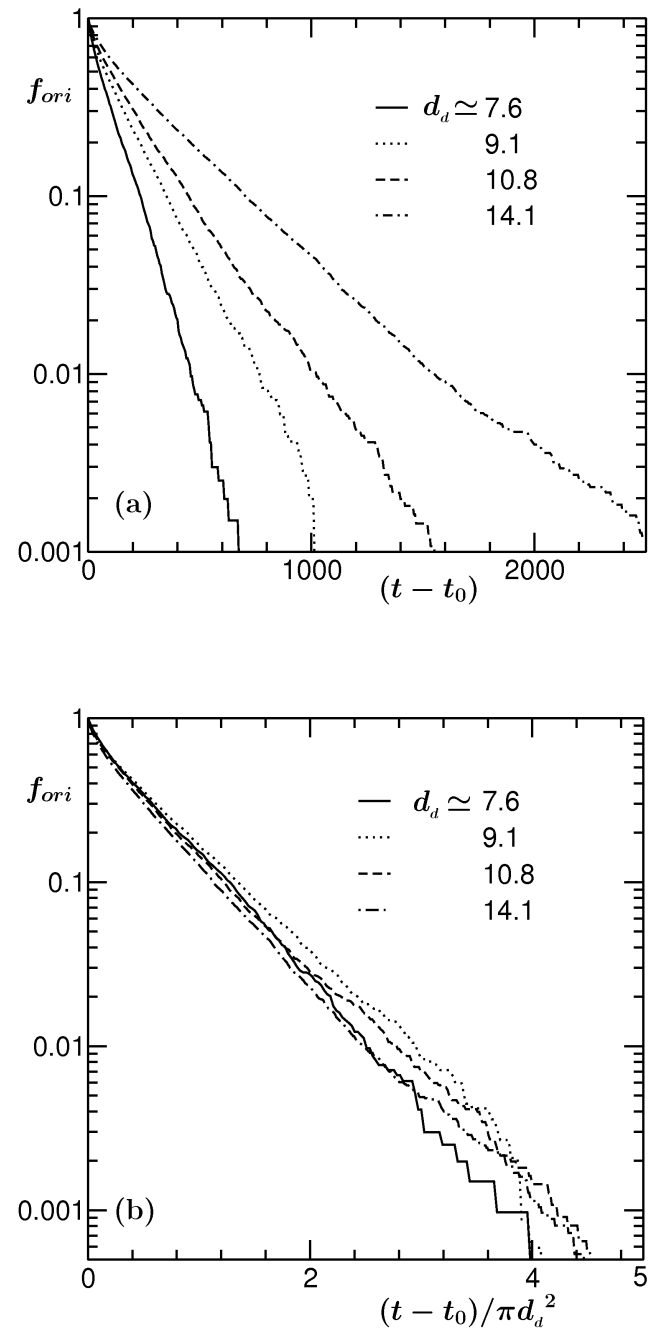

FIG. 10. Same as Fig. 9 but in the binary fluid context.

when the density inside the droplets reached "saturation". First, to rule out the possibility of LS mechanism, we provide the following discussion here.

As we mentioned in the introduction, the LS mechanism of domain growth also leads to an exponent $1 / 3$, same as BS. To distinguish between the two, we studied the droplet growth for the same overall density using AT also. AT being purely stochastic in nature will give rise to the LS mechanism only. For the sake of brevity we do not present the results here. In this case, it appears that the droplets are almost static which is consistent with the LS mechanism where domains grow primarily via the particle concentration diffusion. As we will see below, this is not the case when we use NHT.

To substantiate the above difference further, we investigate if the number of particles in a droplet, before it collides, changes with time, for NHT. In Fig. 6 we show $N_{p}$ in a typical large droplet as a function of time during the period when it does not undergo collision. The nature of the plot is rather flat, within statistical fluctuations. Notice that the period of time over which this plot is shown, the average number of particles in liquid droplets has significantly increased (see Fig. 3) due to collision among other droplets. This confirms that, in this case, 

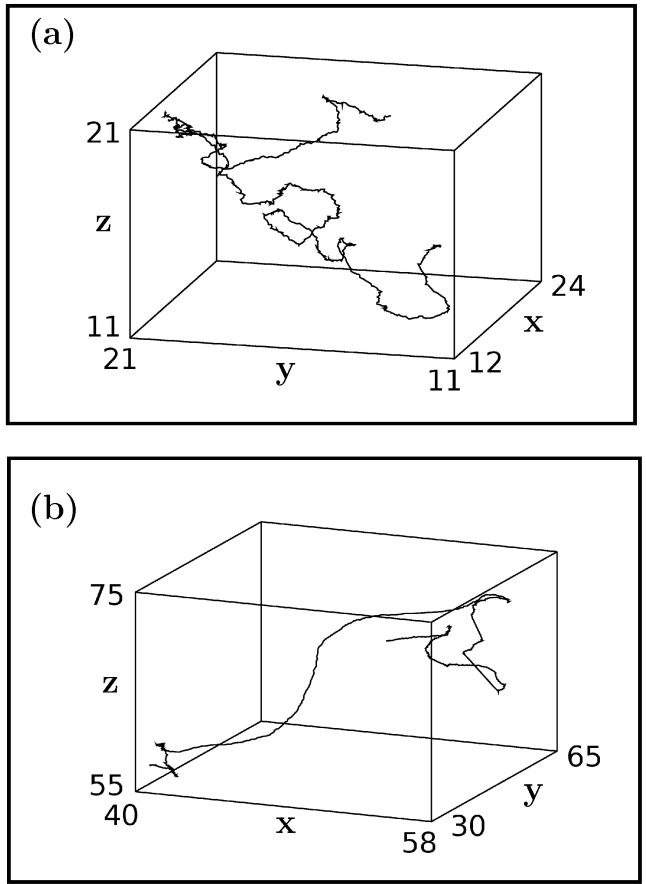

FIG. 11. (a) Trajectory of a typical droplet the system is in equilibrium, i.e., there is only one droplet. The result was obtained using NHT. (b) Same as (a) but in the non-equilibrium situation. In both the cases, only parts of the boxes are shown.

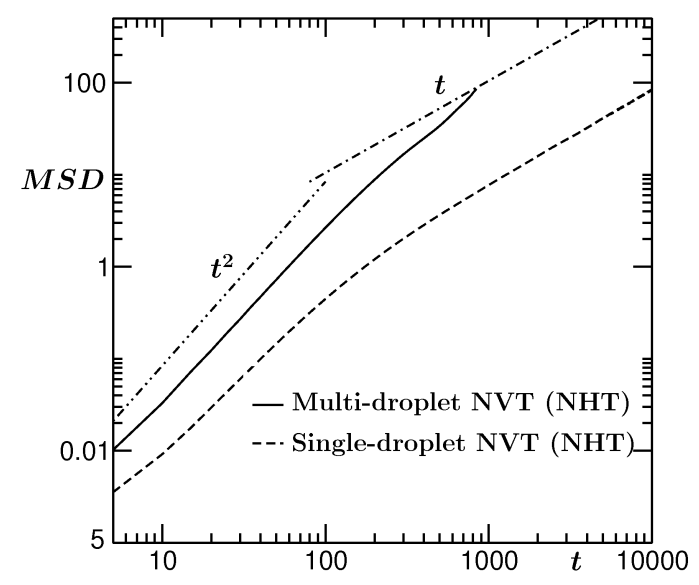

FIG. 12. Log-log plots of the mean squared displacements vs time, of droplets in nonequilibrium (multi-droplet) and equilibrium (single-droplet) situations. Both correspond to an averaging over 6 droplets, obtained using NVT (NHT). Note that in both the cases the droplets are of approximately same size.

the droplets do not grow due to deposition of additional particles onto them via standard diffusion mechanism, as in the LS case. In such a situation, the only way for the droplets to grow must be the collisions when they move.

One possibility for the droplet motion is that the droplets are very compact objects and all the constituent particles move together. However, if this picture is true, keeping the conservation of total momentum in the system, motion of droplets will be constrained if we accept the temperature in the liquid and vapor domains to be
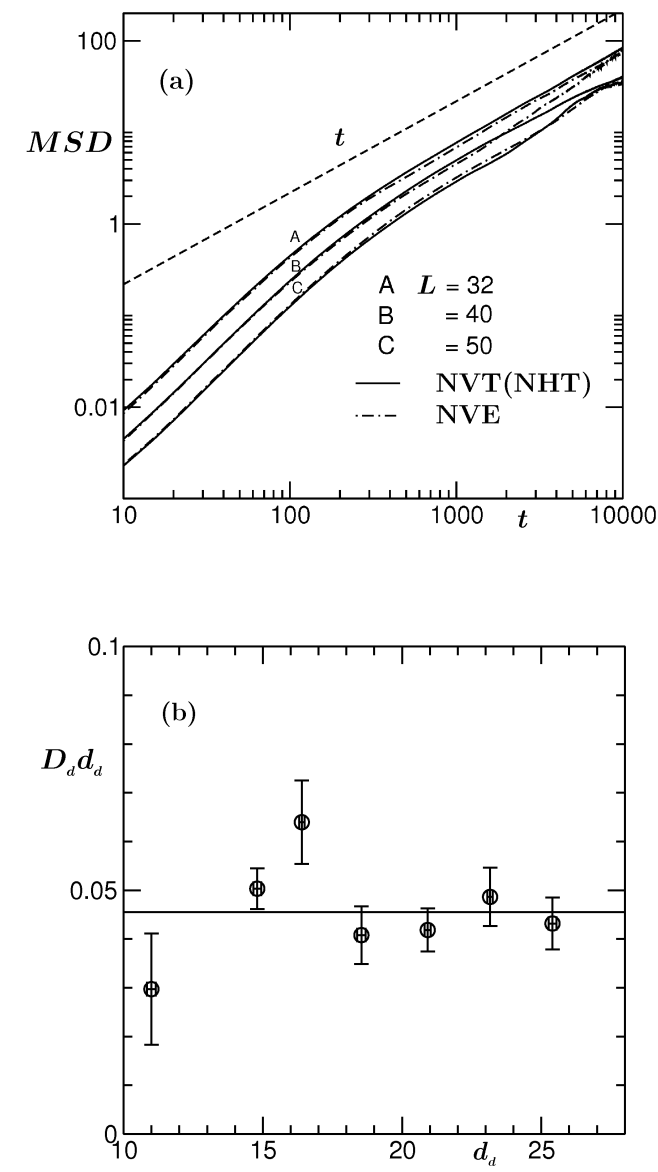

FIG. 13. (a)Log-log plots of the mean squared displacements vs time, of droplets from equilibrium configurations, obtained using NVE as well as NVT (NHT). All results correspond to an averaging over 6 droplets. (b) Plot of $D_{d} d_{d}$ vs the droplet size $d_{d}$, where $D_{d}$ is the droplet diffusivity. The results were obtained in microcanonical ensemble with an averaging over 6 independent initial configurations.

same. But this we discard, following information in Fig. 7 where we plot the fraction, $f_{\text {ori }}$, of original particles at time $t_{0}$, moving with a typical droplet as a function of time translation $t-t_{0}$. It appears that this quantity decays very fast, even though between collisions the size of the droplets remains unchanged. This essentially means that particles from different directions, possibly all, join the droplet to give (possibly) a random directional impact.

Often, in the molecular dynamics simulations in canonical ensemble, it is observed that application of thermostats brings in undesired features. It is, however, understood that a simulation in microcanonical (NVE) ensemble preserves all the requirements of hydrodynamics. But this ensemble, as already stated, is unwanted to study the nonequilibrium dynamics in the two-phase region. Needless to mention, it is a perfect candidate when one has only one droplet in the system, i.e., configurationwise the system has achieved equilibrium. So, it will be a good idea to compare the results of Fig. 7 with the equilibrium NVE MD runs. This, in addition to providing a comparative microscopic picture of droplet motion in equilibrium and nonequilibrium situations, will be a 

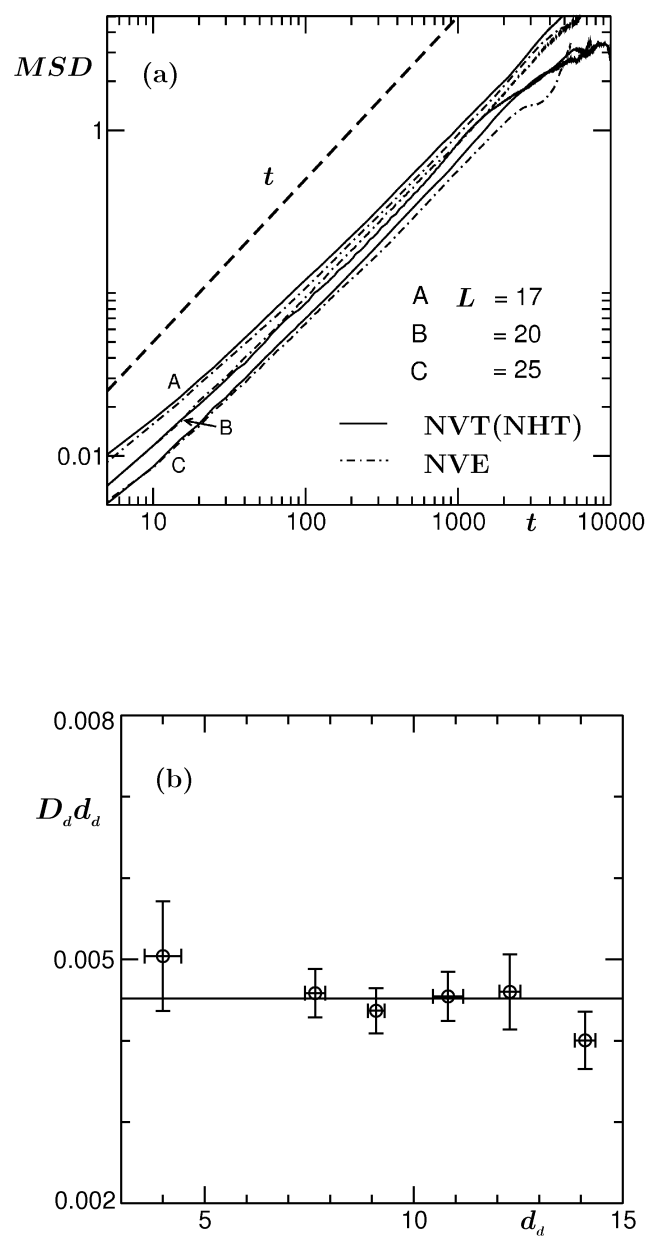

FIG. 14. (a) Log-log plots of the mean squared displacements of equilibrium droplets, from the binary fluid, vs time, for various different system sizes, thus droplet sizes. Results from NVE as well as NVT (NHT) are presented. (b) Plot of $D_{d} d_{d}$ vs the droplet diameter, $d_{d}$, for the binary fluid. Results correspond to an averaging over 8 independent initial configurations in NVE.

good test to confirm the validity of NHT in the study of hydrodynamic phenomena.

In Fig. 8, we present the plots for $f_{\text {ori }}$, on a semi-log plot, for three different cases, viz., equilibrium in NVE, equilibrium with NHT and nonequilibirum with NHT. In all the cases, the droplet sizes are roughly the same. A very nice consistency of the curve for the nonequilibrium situation with NHT with the other ones speaks about the usefulness of NHT in such studies. On the other hand, linear looks of the curves on this semi-log plot is informative of an exponential decay of original particles from the droplet. Note that the system size used for the equilibrium cases is smaller than the nonequilibrium case. This is due to the fact that in equilibrium only one droplet exists and so a smaller system size is a necessity to match the size of the droplet with the nonequilibrium case.

In Fig. 9(a) we plot $f_{\text {ori }}$ vs $t-t_{0}$, for different droplet sizes from equilibrium configurations in NVE ensemble. It appears that the time scale of decay strongly depends on the size of the droplets. Note that the particles leave a droplet from the boundary or surface region. Considering the fact that the quantity of interest here is a volume fraction and further the length dependence of volume to surface ratio, it is expected that the time scale of decay should be longer for larger droplets. It appears that there is nice collapse of data from different droplet radii (see Fig. 9(b)), when the abscissa is scaled by the droplet surface area. According to the above argument, however, the time scale should have a linear dependence upon $d_{d}$. But, we could not yet construct an argument for this quadratic nature which appears to be the fact. Note that the dynamics is rather complex and one needs further probing to understand this behavior appropriately. Similar results from a binary Lennard-Jones fluid are also included in Fig. 10(a) and (b). Here the appropriate quantity is the fraction of original A-particles (the droplets contain A-particles as the majority species) moving with the droplet. The results are obtained from MD simulations in NVE ensemble with equilibrium configurations. Here also the qualitative nature of the decay is same as in the vapor-liquid case.

After the detour on the discussion of compactness of the droplets, we come back to investigate the nature of the droplet motion. In Fig. 11(a) and (b) we show a comparison between trajectories of typical droplets in the equilibrium and nonequilibrium situations from NVT (NHT). In both the cases, droplets are of same size and the trajectories are recorded over the same time length. Both the trajectories look random. However, it is clearly visible that the droplet in the nonequilibrium case spans larger distance than the equilibrium droplet. This is an indication of the presence of inter-droplet interaction in the nonequilibrium (multi-droplet) situation. To further strengthen our claim, in Fig. 12 we plot the droplet MSD vs $t$ for two droplets of same size from equilibrium and nonequilibrium configurations using NHT. While the MSD in the equilibrium case shows linear behavior at late time, a characteristic of the Brownian diffusive motion, the nonequilibrium case does not show this feature. In the equilibrium case, there is only one droplet and so the inter-droplet interaction does not come into the picture, thus the motion is expected to be Brownian. Also, as already claimed, the MSD value for the nonequilibrium situation is higher than the equilibrium case. All these point to the presence of inter-droplet force. Note that in the nonequilibrium case, it is not possible to obtain MSD over an extended period of time because of the finite time available before the droplets collide. Of course, this would be possible in the dilute droplet density limit. But this will require very large system and we do not have possession of enough computational resources that this exercise would demand. We close the discussion on inter-droplet interaction in nonequilibrium situation by stating that our estimate [29] for the amplitude ratios for the growths obtained using NHT and AT gives significantly higher value than the one quoted in Eq. (3) for $A_{B S} / A_{L S}$. This also suggests the presence of this interaction.

Next, to further justify the utility of NHT as a hydrodynamics preserving thermostat, we present results for the MSD in equilibrium from NHT as well as NVE, in Fig. 13(a). In addition to this justification, these results will 
provide important physics information as well. Results for different droplet sizes are plotted, as indicated in the figure. The dashed line here corresponds to the diffusive $(\sim t)$ behavior. Results from NHT are in excellent agreement with NVE. Here we should mention that in our MD simulations in NHT, the temperature was controlled by coupling with the absolute velocities, a standard practice followed. There are more recent algorithms where, instead of the absolute velocities, one uses the relative velocities that provides Galilean invariance [39]. In future, it would be interesting to compare our simple NHT results with the latter to see what further improvement one can bring in.

Next we calculate the droplet diffusivity $D_{d}$, in equilibrium condition, for various different system sizes that automatically leads to different droplet sizes. In Fig. 13(b) we plot $D_{d} d_{d}$ vs the droplet diameter $d_{d}$. Within statistical fluctuation, $D_{d} d_{d}$ remains constant. This validates the SES relation even in such complex droplet diffusion case.

In Fig. 14(a) we plot the MSD of the equilibrium droplets in the binary fluid case for various different system sizes. All of them show the linear diffusive behavior, characteristic of the Brownian motion. Note that unlike the vapor-liquid case, the initial faster $t^{2}$ regime is absent here. This can be attributed to the difference in density of the background fluids in the two cases. In the vaporliquid situation the low density of the background fluid facilitates the ballistic like faster motion of the droplet at early time leading to the $t^{2}$ regime, whereas in the binary fluid case the high density background suppresses it. For the same reason, overall MSD in the binary fluid is much less even though in both the cases results are presented for same time length. Here also we plot the MSD from both NVE and NVT (NHT) ensembles. The results for NVE are again nicely consistent with the NVT results. Exercises in Fig. 13(a) and 14(a) once more confirm the validity of NHT in studying hydrodynamic phenomena. In Fig. 14(b) we plot $D_{d} d_{d}$ vs $d_{d}$ for equilibrium binary fluid. The results are again in agreement with the SES relation.

\section{CONCLUSION}

In conclusion, we have studied the dynamics of droplets during the nonequilibrium evolution of a phase separating fluid [18, 29], being in the nucleation regime of the phase diagram. Molecular dynamics simulations were used where a Nosé-Hoover thermostat controlled the temperature. The average volume of droplets grows linearly with time. It is demonstrated that the droplets grow via collisions. The motion of the droplets is not entirely random because of inter-droplet interaction even for reasonable low droplet density. This information was obtained from comparative study of the droplet dynamics in nonequilibrium situation and the equilibrium case. In the latter case, very similar results from the NVE ensemble molecular dynamics and those from the NVT ensemble with a Nosé-Hoover thermostat confirms the usefulness of this thermostat for the study of hydrodynamic phenomena. In equilibrium, the droplets exhibit Brownian motion and obey the Stokes-Einstein-Sutherland relation. Results for the equilibrium droplet dynamics are presented for a binary fluid [37, 38] as well.

It is observed that the particles in a droplet do not move together even though between collisions the droplets keep their size intact. During the course of the motion, some particles leave and approximately a same number of particles join from various directions. We have found that the fraction of original particles moving with a droplet decays exponentially fast.

In nonequilibrium situation it would be interesting to take a look at the space dependence of temperature. Of particular interest would be to study the temperature profile at the meeting area of two colliding droplets. It is expected that such collisions would give rise to some temperature increase. If so, understanding of how that enhanced temperature decays to the system value with time should also be of significant interest. Study with varying overall density will be of utmost importance to understand the dynamics further. In addition, understanding of decay of autocorrelation functions, probing the aging dynamics [40] in these systems, will be of general interest.

In this paper, the results for the nonequilibrium phenomena are presented only for the vapor-liquid phase separation. Needless to say, a similar study for a binary fluid will be very interesting. This latter problem will, of course, be computationally extremely demanding because of the overall high particle density throughout the system.

\section{ACKNOWLEDGEMENT}

SKD acknowledges discussion with K. Binder and F. Schmid. SKD and SR acknowledge financial support from the Department of Science and Technology, India, via Grant No SR/S2/RJN-13/2009. SR is grateful to the Council of Scientific and Industrial Research, India, for their research fellowship.
[1] Nucleation, edited by A.C. Zettlemoyer (Dekker, New York, 1969).

[2] F.F. Abraham, Homogeneous Nucleation Theory (Academic, New York, 1974).

[3] K. Binder, Rep. Prog. Phys. 50, 783 (1987).
[4] D. Kashchiev, Nucleation: Basic theory with Applications (Butterworth-Heinemann, Oxford, 2000).

[5] K. Binder in Kinetics of Phase Transitions (CRC Press, Boca Raton, 2009), edited by S. Puri and V. Wadhawan.

[6] L.D. Gelb, K.E. Gubbins, R. Radhakrishnan and M. Sliwinska- Bartkowiak, Rep. Prog. Phys. 62, 1573 (1999). 
[7] Handbook of Porous Solids, edited by F. Schüth, K.S.W. Sing, and J. Weitkamp (Wiley-VCH, Weinheim, 2002).

[8] T.M. Squires and S.R. Quake, Rev. Mod. Phys. 77, 977 (2005).

[9] A.J. Bray, Adv. Phys. 51, 481 (2002).

[10] A. Onuki, Phase Transition Dynamics (Cambridge University Press, UK, 2002).

[11] K. Binder, in Phase transformation of Materials, edited by R.W. Cahn, P. Haasen and E.J. Kramer (VCH, Weinheim, 1991), Vol.5, p.405.

[12] R.A.L. Jones, Soft Condensed Matter (Oxford University Press, Oxford, 2008).

[13] I.M. Lifshitz and V.V. Slyozov, J. Phys. Chem. Solids 19, 35 (1961).

[14] S. Majumder and S.K. Das, Phys. Rev. E 81, 050102 (2010).

[15] S. Majumder and S.K. Das, Phys. Rev. E 84, 021110 (2011).

[16] J.-P. Hansen and I.R. McDonald, Theory of Simple Liquids (Academic Press, London, 2008).

[17] S. Ahmad, S.K. Das and S. Puri, Phys. Rev. E. 82, 040107 (2010).

[18] S. Majumder and S.K. Das, Europhys. Lett. 95, 46002 (2011).

[19] H. Furukawa, Phys. Rev. A 31, 1103 (1985).

[20] H. Furukawa, Phys. Rev. A 36, 2288 (1987).

[21] E.D. Siggia, Phys. Rev. A 20, 595 (1979).

[22] K. Binder and D. Stauffer, Phys. Rev. Lett. 33, 1006 (1974).

[23] K. Binder, Phys. Rev. B 15, 4425 (1977).
[24] H. Tanaka, J. Chem. Phys. 105, 10099 (1996).

[25] H. Tanaka, J. Chem. Phys. 107, 3734 (1997).

[26] H. Tanaka, J. Chem. Phys. 103 (6), 2361 (1995)

[27] V. Kumaran, J. Chem. Phys. 109, 7644 (1998).

[28] V. Kumaran, J. Chem. Phys. 112, 10984 (2000).

[29] S. Roy and S.K. Das, Phys. Rev. E 85, 050602 (2012).

[30] F. Perrot, P. Guenoum, T. Baumberger and D. Beysens, Phys. Rev. Lett. 73, 688 (1994).

[31] T.M. Squires and J.F. Brady, Phys. Fluids 17, 073101 (2005).

[32] S.K. Das, J.V. Sengers and M.E. Fisher, J. Chem. Phys. 127, 144506 (2007).

[33] D. Frenkel, B. Smit, Understanding Molecular Simulations: From Algorithm to Applications (Academic Press, San Diego, 2002).

[34] M.P. Allen and D.J. Tildesley, Computer Simulations of Liquids (Clarendon, Oxford, 1987).

[35] W.G. Hoover, Computational Statistical Mechanics, Volume 11 of Studies in Modern Thermodynamics, Elsevier (1991).

[36] S.K. Das and S. Puri, Phys. Rev. E. 65, 026141 (2002).

[37] S.K. Das, J. Horbach, S. Puri, K. Binder, M.E. Fisher and J.V. Sengers, J. Chem. Phys. 125, 024506 (2006).

[38] S. Roy and S.K. Das, Europhys. Lett. 94, 36001 (2011).

[39] M.P. Allen and F. Schmid, Molecular Simulation 33, 21 (2006).

[40] M. Zannetti, in Kinetics of Phase Transitions (CRC Press, Boca Raton, 2009), edited by S. Puri and V. Wadhawan. 